\title{
Mortalidade infantil pós-neonatal e qualidade da assistência médica: um estudo caso-controle
}

\author{
Postneonatal infant mortality and quality of medical assistance: a case-control study
}

\author{
Antônio P. Caldeira' ${ }^{1}$, Elizabeth França ${ }^{2}$, Eugênio M.A. Goulart ${ }^{3}$
}

\section{Resumo}

Objetivo: identificar os fatores de risco para a mortalidade infantil pós-neonatal por diarréia e pneumonia relacionados à qualidade da assistência médica.

Métodos: estudo tipo caso-controle, de base populacional, de 277 óbitos infantis pós-neonatais por diarréia e pneumonia ocorridos na Região Metropolitana de Belo Horizonte, entre maio de 1991 a abril de 1992. Os casos foram comparados com controles hospitalares, emparelhados por patologia, idade e hospital. As informações sobre casos e controles foram coletadas através de prontuários médicos e entrevistas domiciliares, sendo analisadas algumas variáveis relacionadas à qualidade da assistência médica. Utilizou-se o teste de McNemar e o método de regressão logística condicional para a definição dos fatores de risco para o óbito.

Resultados: a regressão logística multivariada mostrou os seguintes fatores independentemente associados a maior risco de óbito pós-neonatal por diarréia e pneumonia: atraso vacinal $(\mathrm{OR}=2,48$; IC95\%=1,17-5,23), estado geral (grave) à admissão hospitalar $(\mathrm{OR}=10,94$; IC95\%=4,91-24,34), não realização de procedimentos hospitalares $(\mathrm{OR}=10,08$; IC95\%=3,55-20,59) e desnutrição presente no momento da internação (OR=3,58; IC95\%=1,42-9,07).

Conclusões: os resultados indicam a baixa qualidade da assistência médica como um importante fator de risco para o óbito infantil pós-neonatal por causas evitáveis. Os autores salientam a falta de integralidade entre as atividades ambulatoriais e hospitalares como importante determinante da baixa qualidade. Sem desconsiderar o papel preponderante das variáveis socioeconômicas, salienta-se a necessidade de uma ampla discussão sobre o desempenho dos serviços de saúde e mortalidade infantil evitável.

J Pediatr (Rio J) 2001; 77 (6): 461-8: mortalidade infantil, mortalidade pós-neonatal, assistência médica.

\begin{abstract}
Objective: to identify the risk factors for postneonatal infant mortality caused by diarrhea and pneumonia in relation to the quality of medical assistance.

Methods: population-based case-control study of 277 postneonatal infant deaths caused by diarrhea and pneumonia occurring in the metropolitan area of Belo Horizonte, Brazil, between May/1991 and April/1992. The cases were compared with hospitalized controls, and matched by pathology, age and hospital. Information on cases and controls were collected from medical records and through home interviews. Some variables related to the quality of medical care were analyzed. McNemar test and conditional logistic regression were used to define the risk factors for postneonatal deaths.

Results: multiple logistic regression analysis showed the following factors independently associated with increased risk of postneonatal death induced by diarrhea and pneumonia: delayed immunization $(\mathrm{OR}=2.48$; 95\% CI=1.17-5.23), general status (serious) on hospital admission $(\mathrm{OR}=10.94 ; 95 \% \mathrm{CI}=4.91-24.34)$, unaccomplished hospital procedures $(\mathrm{OR}=10.08$; 95\%CI $=3.55$ 20.59) and malnutrition on hospital admission ( $\mathrm{OR}=3.58$; 95\% CI=1.42-9.07).

Conclusions: the results indicate the low quality of medical assistance as an important risk factor for avoidable causes of postneonatal deaths. The authors highlight the lack of integration between the outpatient clinic and hospital activities as an important determinant of low quality. It is necessary that the performance of health services and their effect on avoidable infant mortality be widely discussed, also taking into consideration the preponderant role of socioeconomic variables
\end{abstract}

J Pediatr (Rio J) 2001; 77 (6): 461-8: infant mortality, postneonatal mortality, medical assistance.

\section{Introdução}

A mortalidade infantil tem sido freqüentemente apontada como sensível indicador da qualidade de vida de uma determinada população ${ }^{1,2}$. A infância representa, de fato, uma fase particularmente vulnerável da vida, quando os determinantes biológicos do óbito estão fortemente atrela- 
dos às condições externas, sejam socioeconômicas e ambientais (moradia, alimentação, saneamento, higiene e relações familiares), sejam referentes à disponibilidade de serviços de saúde.

A acentuada redução dos índices de mortalidade infantil observada nas últimas décadas em todo o mundo, contudo, não tem sido acompanhada por sensível melhora da qualidade de vida, sobretudo em países em desenvolvimento. Esse fato tem feito com que alguns autores questionem o real valor da taxa de mortalidade infantil como indicador de qualidade de vida, destacando o papel das medidas de saúde pública para a sobrevida infanti ${ }^{3-5}$. Na verdade, o componente pós-neonatal da mortalidade infantil, reconhecidamente mais sensível às intervenções sanitárias, foi o componente que sofreu maior redução nas últimas décadas no Brasil, podendo-se hoje observar para a maioria do país uma frequiência maior de óbitos infantis no período neona$\mathrm{tal}^{2,6}$.

Essa é uma questão bastante complexa. É certo que a mortalidade infantil tem forte vínculo com as condições socioeconômicas ${ }^{1,7}$. Todavia, não se pode negar a influência das medidas de saúde pública que, muitas vezes simples e de baixo custo, foram responsáveis por considerável redução da mortalidade infantil em todo mundo ${ }^{6,8,9}$. A discussão sobre as tendências da mortalidade infantil nos últimos anos e seus determinantes remete aos limites de uma ou de outra intervenção, mas, sobretudo, destaca a questão da qualidade da assistência ${ }^{9-11}$.

Para o Brasil, com níveis ainda elevados em ambos os componentes da mortalidade infantil, maiores inclusive do que os de alguns países da América Latina ${ }^{4}$, compreender melhor o papel das atividades de assistência à saúde no processo de determinação do óbito infantil é uma necessidade e um compromisso ético. A responsabilidade dos serviços de saúde está diretamente vinculada ao conceito de mortes evitáveis, relativamente antigo ${ }^{12}$, mas relativamente pouco explorado pela literatura científica ${ }^{10}$.

Se a mortalidade neonatal tem determinantes de mais difícil intervenção, o mesmo não ocorre com a mortalidade pós-neonatal. Assim, enquanto a maioria dos óbitos pósneonatais for atribuída ao complexo diarréia-pneumoniadesnutrição ${ }^{13-15}$, a evitabilidade de tais óbitos deve ser uma co-responsabilidade irrestrita da assistência médico-hospitalar. Serviços de saúde de boa qualidade, acessíveis e eficazes, se não forem capazes de intervir no processo desencadeante da doença, deveriam sê-los, pelo menos, no reconhecimento precoce e tratamento adequado ${ }^{10,12}$.

Para a Região Metropolitana de Belo Horizonte, a atenção à criança doente já foi previamente questionada como possível determinante do óbito infantil por França ${ }^{16}$, que observou em seu estudo uma elevada e inesperada proporção de óbitos domiciliares e uma elevada proporção de crianças que evoluíram para o óbito logo após a alta hospitalar. Tais destaques remetem à discussão sobre a acessibilidade e os cuidados da atenção hospitalar. O presente estudo tem como objetivo identificar alguns determinantes da mortalidade infantil pós-neonatal por diarréia e pneumonia associados à qualidade dos serviços de saúde, procurando identificar aspectos da estrutura organizacional e do processo de assistência médica que possam estar envolvidos com o óbito infantil evitável.

\section{Métodos}

O presente trabalho é parte de um estudo sobre determinantes da mortalidade infantil, cuja área analisada foi a Região Metropolitana de Belo Horizonte (RMBH), Minas Gerais ${ }^{16}$. A coleta de dados foi realizada em 13 municípios, além da capital, compreendendo uma população total de aproximadamente 3.360 .000 habitantes, sendo $2 \%$ a proporção de menores de um ano.

Foram analisadas todas as declarações de óbitos pósneonatais referentes a crianças residentes na RMBH no período de maio/1991 a abril/1992. Inicialmente selecionou-se para investigação todas as declarações de óbito com citação de diarréia, pneumonia ou desnutrição, além de outras com citações que após investigação pudessem se enquadrar como tal (anemias, septicemia, desidratação, etc.). Nessa etapa, implantou-se uma busca ativa semanal de óbitos infantis na Secretaria de Estado de Planejamento de Minas Gerais, responsável pela coleta dos óbitos registrados em cartórios. Após investigação hospitalar (revisão de prontuários) e/ou domiciliar (entrevista com a família), optava-se pela inclusão ou exclusão de cada óbito no estudo, conforme se encaixasse ou não no critério de caso. A definição da causa do óbito foi realizada por um médico supervisor do trabalho de campo, após discussão com o responsável pela coleta de dados, e posteriormente confirmada numa revisão por dois outros médicos pediatras de forma independente. Não foi realizada avaliação de concordância. Entretanto, a proporção de desacordo foi mínima e, quando presente, a coordenação da pesquisa tomava a decisão final. Foram excluídos os óbitos associados a malformações congênitas importantes, paralisia cerebral, causas perinatais ou prematuridade extrema, e os óbitos cuja investigação hospitalare domiciliar mostrou-se inconclusiva. Também não foram incluídos nessa análise os óbitos cujo local de ocorrência foi domiciliar ou a via pública, ou aqueles cuja permanência hospitalar imediatamente anterior ao momento do óbito foi muito curta (apenas algumas horas), pois a avaliação da assistência médica proposta se aplica também aos cuidados hospitalares.

$\mathrm{Na}$ investigação hospitalar foram coletadas informações relativas às condições clínicas da criança, procedimentos adotados e condições de acesso aos serviços de saúde até o momento da internação, quando disponíveis no prontuário. No questionário domiciliar, foram levantadas informações relativas às condições socioeconômicas e relacionadas à família, condições ambientais e relacionadas à moradia, condições de gestação e de parto, condições de saúde da criança e evolução do processo de doença que 
gerou a hospitalização. No presente estudo, foram utilizadas as informações pertinentes ao acesso aos serviços de saúde e à qualidade da atenção médico-hospitalar. Incluiuse ainda entre as variáveis estudadas o peso de nascimento, por entender-se que este representa uma medida resumo dos cuidados pré-natais ${ }^{17}$, e o estado nutricional à admissão através da relação peso-idade, porque pode ser compreendido como um indicador de assistência médica continuada, mais do que um mero indicador de acesso ao alimento ou de boas condições de moradia ${ }^{18}$.

Foram definidos como casos os óbitos pós-neonatais (idade de 28 dias a menos de um ano) por diarréia (diarréia como quadro clínico predominante e antecedente a outras patologias, caracterizada pela história clínica) ou pneumonia (pneumonia como quadro clínico predominante, antecedente a outras patologias intercorrentes, confirmada por radiografia de tórax). Para cada caso estudado, localizou-se um controle, representado por criança com mesma patologia, com diferença de idade de até dois meses, internada no mesmo hospital, no mesmo período de ocorrência, que não houvesse evoluído para o óbito (nem durante a internação, nem durante o período de duração do estudo).

Foram considerados como não localizados os prontuários de casos e controles não encontrados em pelo menos duas visitas hospitalares, e os endereços dos familiares que não foram confirmados em pelo menos duas tentativas de localização (ou quando confirmados, não se localizou a família para entrevista). Prontuários não localizados implicavam em perda completa do caso (48 crianças não foram investigadas por esse motivo), entretanto a falta da entrevista domiciliar não era considerada como um critério de exclusão.

Para a operacionalização de todas as etapas da coleta de dados, contou-se com equipes especialmente treinadas (estudantes de Medicina para a investigação hospitalar e estudantes de Ciências Sociais para a entrevista domiciliar). Realizou-se previamente um estudo piloto, testando na prática o questionário e a equipe de entrevistadores. Foram realizadas, também, revisitas a algumas famílias, como forma de controle de qualidade das informações e para detectar possíveis vieses (5\% das entrevistas dos seis primeiros meses da pesquisa, selecionadas por amostragem aleatória).

Para acesso aos prontuários, foram obtidos previamente apoio e consentimento dos hospitais, assegurando-se total sigilo das informações coletadas. Para a entrevista domiciliar, solicitou-se consentimento formal da família, através de carta de apresentação dos entrevistadores, assegurandose, igualmente, a confidencialidade das informações.

As análises estatísticas foram realizadas a partir do programa Epi Info ${ }^{19}$, admitindo-se um nível de significância de 5\% nos testes estatísticos e utilizando-se a Razão de Odds (RO) e o intervalo de confiança a $95 \%$ para aferição das variáveis associadas ao óbito. A regressão logística múltipla foi empregada através do programa Multlr ${ }^{20}$ para definição das variáveis que, numa análise estatística conjunta, pudessem apresentar efeito explicativo independente.

O presente trabalho teve sua execução aprovada pelo Comitê de Ética da Faculdade de Medicina da UFMG (1988) e pelo Comitê de Ética da OPAS (1989), e contou com o apoio financeiro da Organização Pan-Americana de Saúde (OPAS) e da Fundação do Amparo à Pesquisa de Minas Gerais (FAPEMIG).

\section{Resultados}

Durante o estudo, foram selecionadas para investigação 665 óbitos pós-neonatais. Após investigação hospitalar, foram excluídos 154 óbitos que se mostraram referentes a outras patologias $(n=35)$, eram associados a malformações congênitas importantes $(\mathrm{n}=32)$ ou apresentavam história inconclusiva $(n=47)$. Excluíram-se, também, óbitos referente a gêmeos (optando-se por incluir apenas um dos irmãos; $n=6$ ) e crianças institucionalizadas ou não residentes na RMBH ou cuja seleção inicial foi incorreta $(n=34)$. Entre os 511 óbitos então selecionados, foram ainda excluídos deste estudo os óbitos domiciliares (136 óbitos). Para 50 óbitos, a análise detalhada dos mesmos revelou tratarem-se de casos com patologias graves associadas (por exemplo, meningite bacteriana) ou verificou-se que o emparelhamento não era satisfatório. Foram perdidos 48 casos por não ter sido possível localizar o prontuário no hospital. Assim, o presente estudo refere-se à análise de 277 pares.

A entrevista domiciliar (com a mãe ou responsável) foi obtida para 240 casos, o que permitiu a confirmação da maioria das informações coletadas no hospital e complementação das informações ausentes no prontuário. Para os controles, a entrevista domiciliar foi obtida de 139 famílias (por questões logísticas, foi definido que as entrevistas domiciliares para os controles seria realizada apenas nos seis primeiros meses).

A distribuição dos óbitos estudados entre os hospitais ocorreu da seguinte forma: $80,1 \%$ em hospitais privados, contratados pelo Sistema Único de Saúde (SUS), 14,8\% em hospitais públicos, e 5,1\% em hospitais filantrópicos.

A idade média das crianças que evoluíram para óbito foi de 4,9 meses, com mediana de 4,0 meses. Para as crianças estudadas como controles, a idade média foi de 4,8 meses, com mediana também de 4,0 meses. Foram estudados 143 pares de crianças cuja patologia básica foi considerada diarréia (casos e controles) e 134 pares de crianças, em cada grupo, cuja patologia básica era pneumonia. Em ambos os grupos, observou-se predomínio do sexo masculino $(66,8 \%$ dos casos e $62,5 \%$ dos controles).

A duração da doença antes da hospitalização (a partir da percepção da mãe) foi semelhante para ambos os grupos (mediana de três dias para casos e controles e médias de 6,0 e 6,3 dias, respectivamente; $p=0,709)$. A permanência 
hospitalar apresentou uma mediana semelhante para casos e controles (sete dias), embora a duração média tenha se mostrado maior para as crianças que evoluíram para o óbito (média de 11,4 dias para os casos e 9,1 dias para os controles; $\mathrm{p}=0,008$ ).

Os dados referentes à utilização de medicamentos e realização de exames laboratoriais permitiram constatar uma maior utilização de antimicrobianos de amplo espectro pelo grupo dos casos e, de modo similar, maior realização de exames laboratoriais para esse grupo. O exame laboratorial mais solicitado foi o hemograma $(76,9 \%$ dos casos e $62,1 \%$ dos controles). Para poucos pacientes, foram realizados exames microbiológicos (28,2\% dos casos e $15,2 \%$ dos controles).

Em relação à dieta prescrita no momento da internação e à dieta predominantemente utilizada durante a permanência hospitalar, as informações são apresentadas na Tabela 1. Tanto para casos como para controles, o principal critério para a não utilização da dieta (dieta zero) foi a prescrição médica, e não a recusa por parte do paciente. O tempo de dieta zero foi significativamente maior para casos do que para controles (médias de 21,6 horas e 14,8 horas, respectivamente). A análise particularizada para as crianças cuja patologia básica era diarréia mostra dados semelhantes, os casos também foram mantidos por tempo significativamente maior em regime de dieta suspensa (média de 23,5 horas para os casos e 14,5 horas para os controles), principalmente por recomendação médica.

A Tabela 2 apresenta o resultado da análise bivariada para algumas variáveis relacionadas à qualidade da assistência para casos e controles, com dados analisados de forma emparelhada. Para algumas variáveis, não foi possível obter a informação nem no prontuário médico, nem com a mãe (entrevista domiciliar), resultando em menor número de pares para a análise. Nesta primeira etapa, mostraram-se significantes: o relato de assistência médica prévia para a patologia que gerou a hospitalização, a história de internação prévia, a constatação de atraso vacinal, o fato de não ter recebido o BCG, a não realização de algum procedimento hospitalar, o estado geral grave à internação e a desnutrição presente no momento da internação.

Em relação às variáveis apresentadas na Tabela 2, são importantes alguns destaques. Cerca de $51,3 \%$ dos casos e $38,5 \%$ dos controles tiveram assistência médica prévia à internação. Tanto para casos como para controles, os serviços mais procurados foram os ambulatórios hospitalares ou pronto-socorros $(75,8 \%$ e $73,3 \%$, respectivamente), enquanto os centros de saúde foram pouco acessados $(9,7 \%$ dos casos e 14,9\% dos controles). Outros serviços (consultórios particulares, clínicas, transferências de outros hospitais, etc.) também foram referidos $(14,5 \%$ dos casos e $11,8 \%$ dos controles).

Os procedimentos hospitalares não realizados (registrados nos prontuários médicos) mais observados foram: exames laboratoriais e radiológicos $(45,1 \%)$; admissões em CTI (25,0\%); intervenções cirúrgicas, tais como punções, drenagens e dissecções venosas (15,0\%); administração de medicamentos (principalmente antibióticos) ou hemoderivados $(10,2 \%)$ e outros, como fisioterapia, outros exames ou intervenções $(4,7 \%)$.

Para a análise multivariada, foram incluídas todas as variáveis que na análise bivariada apresentavam um valor de $p$ inferior a 0,20. Após ajustamento, as variáveis que mostraram, de modo independente, maior associação com o óbito infantil pós-neonatal, foram: histórico de atraso vacinal, estado geral grave no momento da internação, procedimento hospitalar não realizado e desnutrição presente no momento da internação (Tabela 3).

Tabela 1 - Principais tipos de dietas prescritas e a proporção de sua utilização para casos e controles, Região Metropolitana de Belo Horizonte, maio/91 a abril/92

\begin{tabular}{lcccc}
\hline Tipos de Dieta & \multicolumn{2}{c}{ Casos } & \multicolumn{2}{c}{ Controles } \\
& $\mathbf{n}$ & $\mathbf{\%}$ & $\mathbf{n}$ & $\mathbf{\%}$ \\
\hline Dieta prescrita à admissão: & & & & \\
$\quad$ Dieta suspensa ou Dieta zero & 112 & 40,4 & 83 & 30,0 \\
$\quad$ Leite materno & 3 & 1,1 & 13 & 4,7 \\
$\quad$ Dieta livre para a idade & 53 & 19,1 & 83 & 30,0 \\
$\quad$ Restrita & 97 & 35,0 & 96 & 34,7 \\
$\quad$ Outras & 12 & 4,3 & 2 & 0,7 \\
Dieta predominante durante internação: & & & & \\
$\quad$ Dieta suspensa ou Dieta zero & 52 & 36,4 & 1 & 0,7 \\
$\quad$ Leite materno & 2 & 1,5 & 7 & 4,9 \\
$\quad$ Dieta livre para a idade & 23 & 16,1 & 65 & 45,5 \\
$\quad$ Restrita & 52 & 36,4 & 69 & 48,7 \\
$\quad$ Outras & 13 & 9,2 & 2 & 1,5 \\
\hline
\end{tabular}


Tabela 2 - Análise bivariada para algumas variáveis relacionadas à qualidade da assistência médica e mortalidade infantil pós-neonatal (casos e controles emparelhados) na Região Metropolitana de Belo Horizonte, maio/91 a abril/92

\begin{tabular}{|c|c|c|c|c|c|}
\hline Variável & Casos & Controles & $\mathbf{p}$ & RO* & IC95\% \\
\hline \multicolumn{6}{|c|}{ Antecedentes à doença atual } \\
\hline \multicolumn{6}{|c|}{ Peso ao nascer } \\
\hline$<2500 \mathrm{~g}$ & 71 & 54 & 0,086 & 1,49 & $0,95-2,33$ \\
\hline$\geq 2500 \mathrm{~g}$ & 151 & 168 & & & \\
\hline \multicolumn{6}{|l|}{ Atraso vacinal } \\
\hline Sim & 109 & 58 & 0,001 & 2,96 & $1,86-4,74$ \\
\hline Não & 128 & 179 & & & \\
\hline \multicolumn{6}{|l|}{ Antecedente BCG } \\
\hline Não & 79 & 35 & 0,001 & 3,20 & $1,89-5,46$ \\
\hline Sim & 150 & 194 & & & \\
\hline \multicolumn{6}{|l|}{ Internação prévia } \\
\hline $\operatorname{Sim}^{3}$ & 126 & 97 & 0,009 & 1,66 & $1,12-2,45$ \\
\hline Não & 106 & 135 & & & \\
\hline \multicolumn{6}{|l|}{ Peso à internação } \\
\hline$<-2,0$ escore $\mathrm{Z}$ & 152 & 94 & 0,001 & 5,83 & $3,07-11,32$ \\
\hline$\geq-2,0$ escore $\mathrm{Z}$ & 91 & 149 & & & \\
\hline \multicolumn{6}{|c|}{ Associados à doença atual } \\
\hline \multicolumn{6}{|c|}{ Assist. médica prévia à internação atual } \\
\hline Sim & 96 & 72 & 0,009 & 1,89 & $1,16-3,09$ \\
\hline Não & 91 & 115 & & & \\
\hline \multicolumn{6}{|c|}{ Horário de hospitalização } \\
\hline Noturno & 76 & 73 & 0,845 & 1,06 & $0,71-1,58$ \\
\hline Diurno & 154 & 157 & & & \\
\hline \multicolumn{6}{|l|}{ Dia de internação } \\
\hline Fim-de-semana & 65 & 67 & 0,918 & 1,04 & $0,68-1,59$ \\
\hline Dia útil & 212 & 210 & & & \\
\hline \multicolumn{6}{|c|}{ Estado geral à internação } \\
\hline Grave & 207 & 72 & 0,001 & 10,64 & $6,01-19,20$ \\
\hline Moderado/ bom & 70 & 205 & & & \\
\hline \multicolumn{6}{|c|}{ Procedimento hospitalar não realizado } \\
\hline Sim & 74 & 9 & 0,001 & 9,13 & $4,25-20,43$ \\
\hline Não & 203 & 268 & & & \\
\hline
\end{tabular}

(*) Razão de Odds

\section{Discussão}

O conjunto de determinantes do óbito infantilé bastante amplo e complexo, existindo entre esses determinantes uma rede de interações que precisa ser melhor conhecida. O presente trabalho procurou analisar algumas variáveis pouco estudadas, referentes à qualidade da assistência. Estudos semelhantes têm o mérito de redimensionar o papel dos serviços de saúde na evitabilidade do óbito infantil, apontando a co-responsabilidade do atendimento ambulatorial e hospitalar e acusando a necessidade de intervenções setoriais específicas ${ }^{10}$. A evitabilidade dos óbitos infantis por diarréia e pneumonia já tem sido salientada há tempos, sendo mesmo considerados eventos "sentinelas" de alguma falha do sistema de saúde ${ }^{12}$. Medidas oportunas e adequadas em serviços de saúde, facilmente disponíveis com acesso universal, são pressupostos de boa qualidade, passíveis de intervir favoravelmente no processo de mortalidade infantil.

O delineamento empregado neste trabalho, tipo casocontrole, com análise de prontuários e entrevista domiciliar, possibilitou uma maior confiabilidade em relação à definição da causa básica dos óbitos e em relação aos fatores analisados como variáveis determinantes. Particularmente para a RMBH, onde o sub-registro de óbitos é pouco importante ${ }^{21}$, as declarações de óbito podem ser consideradas fontes confiáveis do ponto de vista quantitativo.

O emparelhamento empregado no presente estudo representou uma forma de eliminar, a priori, possíveis fatores de confusão. O emparelhamento por patologia impediu, por 
Tabela 3 - Resultado da análise multivariada* para alguns fatores relacionados à qualidade da assistência médica e mortalidade pós-neonatal, Região Metropolitana de Belo Horizonte, maio/91 a abril/92

\begin{tabular}{|c|c|c|c|}
\hline Variável & $\mathbf{p}$ & $\begin{array}{c}\text { Razão } \\
\text { de Odds }\end{array}$ & IC $95 \%$ \\
\hline Atraso vacinal & 0,01 & 2,48 & $1,17-5,23$ \\
\hline $\begin{array}{l}\text { Estado geral (grave) } \\
\text { à internação }\end{array}$ & 0,0001 & 10,94 & $4,91-24,34$ \\
\hline $\begin{array}{l}\text { Procedimento hospitalar } \\
\text { não realizado }\end{array}$ & 0,0001 & 10,08 & $3,55-20,59$ \\
\hline $\begin{array}{l}\text { Desnutrição presente } \\
\text { à internação }\end{array}$ & 0,007 & 3,58 & $1,42-9,07$ \\
\hline
\end{tabular}

exemplo, que um quadro de diarréia, reconhecidamente um processo quase sempre autolimitado, fosse comparado a uma pneumonia. De forma similar, foi o emparelhamento por idade, já que lactentes jovens são sabidamente mais vulneráveis. Em relação ao emparelhamento por hospitais, este teve como principal objetivo evitar que diferentes estruturas hospitalares fossem analisadas em conjunto. Tantas restrições possivelmente tornaram casos e controles muito semelhantes. Esse superemparelhamento (overmatching) pode ter prejudicado a análise aqui apresentada, seja através da redução do poder estatístico por aumento das perdas, ou impedindo que alguma variável se mostrasse significativa por estar intimamente relacionada com aquelas utilizadas nos critérios de emparelhamento. Na verdade, a opção por um estudo tipo caso-controle para a mortalidade infantil, com controles hospitalares (emparelhados por unidade hospitalar), já representa uma restrição à sua validade externa, pois teoricamente apenas um grupo social estaria sendo analisado, a clientela do hospital. De outra forma, contudo, não seria possível uma análise que priorizasse a qualidade da assistência, com enfoque particular para a qualidade do processo de assistência médica. Tal análise está em concordância com a clássica proposta de Donabedian, que sugere uma avaliação qualitativa do cuidado médico através de três componentes: estrutura, processo e resultado ${ }^{22}$.

Os resultados permitem inferir que os casos chegaram mais graves ao hospital e mantiveram-se mais graves, pois a proporção de antimicrobianos de amplo espectro ou utilizados em patologias mais graves foi maior para o grupo de crianças que evoluíram para o óbito. Por outro lado, a proporção de utilização de penicilinas foi maior para o grupo controle. Foi semelhante a utilização de broncodilatadores para casos e controles, o que indica que a associação com o broncoespasmo não foi fator determinante da gravidade ou do risco.

Foram observadas diferenças também na abordagem laboratorial para casos e controles. Assume-se que a maior proporção de exames como ionogramas, exames do liquor e gasometrias entre os casos seja devida à maior gravidade dos mesmos. Entretanto, nem mesmo o exame laboratorial mais realizado, o hemograma, foi realizado para todos os casos e controles. Embora não seja uma necessidade absoluta, é presumível e justificável sua realização naqueles pacientes para os quais se indicou uma internação hospitalar. Destaca-se, ainda, o baixo número de exames microbiológicos, particularmente tratando-se de patologias infecciosas.

Os dados referentes à dieta prescrita e utilizada para casos e controles são de difícil interpretação. Usualmente para crianças com doença diarréica, o tratamento dietético deve ser individualizado ${ }^{23,24}$, enquanto pacientes com pneumonia devem ter suas dietas suspensas apenas se as condições clínicas não permitirem ${ }^{25}$, o que nem sempre é possível inferir a partir das informações do prontuário. É possível que a maior proporção de casos com "dieta zero" ou "dieta suspensa" seja também um indicador de maior gravidade entre os mesmos.

É notória a mínima proporção de casos e controles que fazia uso de leite materno. Destaca-se também a elevada proporção de prescrição "dieta livre para a idade", já que pode estar implícito aí um certo descaso com aspectos nutricionais no paciente internado. Esse fato merece inclusive análises posteriores, considerando-se que a permanência hospitalar representa uma oportunidade de aprendizagem para a mãe em relação a todos os cuidados com seu filho, inclusive em relação à alimentação.

Entre as variáveis estudadas, mostraram-se associadas ao óbito pós-neonatal por diarréia e pneumonia após a análise multivariada: o estado geral grave à internação, o atraso vacinal, a não realização de procedimento em nível hospitalar e a desnutrição presente à internação. $\mathrm{O}$ baixo peso ao nascer, reconhecidamente associado a maior risco de morte no primeiro ano de vida ${ }^{7,26}$, não se mostrou associado neste estudo. Isso pode ser explicado pelo número de perdas ocorridas para essa informação em particular, pois a mesma só era disponível de modo confiável para 222 pares, o que representou uma perda de quase $20 \%$ !

A variável "estado geral grave à internação" já foi apontada em estudo prévio como fator prognóstico para maior letalidade em crianças menores de um ano com diarréia e pneumonia ${ }^{27}$. Os autores salientaram que, embora se tratasse de uma situação óbvia, merecia considerações posteriores e sugeriram alguns mecanismos para explicar o fato: a) a família não percebe a gravidade do quadro e não procura ajuda; b) a família percebe, procura ajuda, mas não consegue (dificuldade de acesso); c) a criança consegue chegar ao médico e esse não avalia corretamente o quadro e não encaminha para internação; d) a criança tem outros fatores subjacentes (peso baixo, desnutrição, outras doenças associadas) que fariam a patologia agravar rapidamente. $\mathrm{O}$ presente trabalho mostrou que, pelo menos para as patologias mais prevalentes para o óbito infantil pós-neonatal, as mães são capazes de reconhecer precocemente a 
necessidade de intervenção médica, pois a duração média da doença é semelhante para casos e controles (a partir do reconhecimento da mãe até o momento da hospitalização). Também ficou claro que o acesso para o atendimento médico "curativo" (hospitais) não é um fator determinante do óbito, pois as crianças que evoluíram para o óbito tiveram, inclusive, mais assistência do que as crianças do grupo controle. Esse fato aponta para a terceira alternativa, como a mais provável, de que as crianças chegam até o serviço médico, mas não recebem atenção devida, pois não têm suas necessidades atendidas ou seus riscos antecipados. Em relação aos "fatores subjacentes", o presente estudo destacou que pelo menos a desnutrição é uma variável associada de grande relevância, mas atua de forma independente, pois manteve-se no modelo final. Entretanto, a desnutrição ou qualquer outro fator subjacente identificável no momento da consulta médica deveria justificar uma intervenção ainda mais precoce, fato que salienta ainda mais a questão da qualidade da assistência médica (presume-se que justamente as crianças que mais requerem cuidados estariam sendo negligenciadas). Este resultado remete à discussão sobre necessidade, demanda e oferta e à "lei do cuidado inverso", que destaca a distribuição desigual do cuidado médico, inversamente proporcional à necessidade da população assistida ${ }^{28}$. Entende-se que as necessidades são relativas às prioridades e se expressam como demanda se existe disponibilidade de serviços. Se o serviço disponível, todavia, não é efetivo (de boa qualidade), não atua como estratégia de intervenção sanitária e acaba por não responder às necessidades/demandas da população de referência. Essa falta de resposta muitas vezes está na precária organização e articulação da rede de serviços, e tem prejuízos óbvios e desastrosos.

A maioria das famílias que procuraram atendimento médico previamente à internação o fez em hospitais. Freqüentemente, esse tipo de atendimento é executado de forma pontual, desarticulada do processo de assistência médica como um todo ${ }^{29,30}$. Não há dúvidas de que esse tipo de cuidado está em desacordo com o conceito de integralidade, e representa uma quebra do compromisso hierárquico que pressupõe o modelo assistencial. Se essa assistência representa uma opção familiar, pode indicar maior resolutividade dos hospitais em relação aos centros de atendimento básico (pelo menos para os usuários) e/ou dificuldade de acesso à rede básica.

No presente trabalho, o atraso vacinal observado como fator associado ao óbito, mesmo após análise multivariada, deve ser considerado como um indicador de acesso aos centros de saúde. $\mathrm{O}$ acesso tem sido considerado como um dos principais componentes da qualidade da atenção médi$\mathrm{ca}^{31,32}$. Implícito no conceito de acesso, está não somente a disponibilidade geográfica e organizacional das ações e serviços de saúde, mas também a aceitabilidade de tais procedimentos, o que depende da valorização por parte do usuário $^{31}$. Para o atendimento à criança, essa valorização está relacionada a uma atenção integral, que responda aos anseios da família, que represente um apoio facilmente disponível e uma fonte de aprendizagem para lidar com as particularidades de um ser em formação. Negligenciandose tais aspectos, é difícil assimilar a confiança da família. Por outro lado, as unidades básicas freqüentemente são desarticuladas de centros de maior complexidade, e dispõem de poucos (quando dispõem) recursos propedêuticos. Assim, o atraso vacinal destaca tanto a indisponibilidade do serviço como a oportunidade perdida pela unidade básica através de um atendimento não integral, além de sinalizar também com a resposta "desacreditada" da população, que tem preferência pelo atendimento nos ambulatórios e pronto-socorros dos hospitais.

Em relação aos cuidados intra-hospitalares, foi possível perceber que ter algum procedimento não realizado foi fator determinante para predizer o óbito. Os principais procedimentos não realizados ressaltam uma deficiência em termos de estrutura, que tanto pode estar relacionada a aspectos materiais, quanto organizacionais. Esse fato depõe contra a qualidade da assistência. Por outro lado, é possível inferir que, embora a admissão em CTI tenha se mostrado como um procedimento não realizado para apenas $25 \%$ dos casos, esse percentual subestima a necessidade real. Particularmente se observamos que $35 \%$ dos casos não tinham registro de qualquer assistência no momento do óbito, com ou sem manobras de ressuscitação!

Finalmente, outro fator apontado como determinante do óbito foi a desnutrição presente no momento da internação. Essa variável já foi apontada anteriormente por Post e colaboradores $^{27}$, que incluíram uma breve discussão sobre possíveis vieses, a desidratação associada e o fenômeno de "causalidade reversa". Como o critério de aferição foi através da relação peso/idade, esta poderia estar falseada pela desidratação presente no momento da internação, entretanto, os dados foram previamente ajustados pelo grau de hidratação. A causalidade reversa representa um viés temporal que estaria presente se a desnutrição no momento da internação fosse, na verdade, decorrente de uma patologia mais grave e/ou de maior duração. Para este estudo, essa possibilidade foi afastada, considerando-se que a duração média da doença foi semelhante para casos e controles, e que o critério de elegibilidade procurou excluir patologias graves associadas para ambos os grupos.

Um crescimento adequado representa, provavelmente, de forma isolada, o melhor indicador de bem-estar infantil. A desnutrição protéico-calórica, geralmente aferida pela relação peso/idade, representa um comprometimento evolutivo do processo de crescimento. Devido ao potencial sinergismo entre as doenças infecciosas e a desnutrição infantil, esta é naturalmente associada à mortalidade infantil, sendo reconhecidamente o maior problema de saúde pública para os países em desenvolvimento ${ }^{18}$. Essa associação não deveria ser traduzida no simples reconhecimento da desnutrição como uma "patologia social". Deveria ser compromisso dos serviços de saúde antecipar medidas de recuperação nutricional e assistência oportuna, evitando 
complicações de patologias comuns, como a diarréia e a pneumonia. Assume maior gravidade o fato do ponto de corte utilizado neste estudo, de 2 escores Z, representar uma desnutrição grave. Isto significa que possivelmente formas leves e moderadas da desnutrição infantil também sejam negligenciadas.

Concluindo, a grande relevância deste estudo foi apontar, de forma precisa, a baixa qualidade do cuidado médico (tanto em âmbito assistencial como organizacional) como um fator determinante da mortalidade infantil pós-neonatal, referendando o pensamento das famílias que sofrem a perda das crianças ${ }^{33}$. Está além dos objetivos do presente texto uma discussão mais profunda sobre aspectos organizacionais da rede de assistência à saúde, mas pretende-se que possa despertar a autocrítica de prestadores e gestores, trazendo a responsabilidade do óbito infantil para dentro dos serviços de saúde ("evento-sentinela"). Destaca-se que essa pretensão não implica em negação dos determinantes sociais, culturais e econômicos da mortalidade infantil, mas sim no comprometimento dos serviços de saúde com assistência adequada e de boa qualidade.

\section{Referências bibliográficas}

1. Paim JS, Dias CN, Araújo JD. Influência de fatores sociais e ambientais na mortalidade infantil. Bol of Sanit Panam 1980; 84: $327-40$.

2. Szwarcwald CL, Leal MC, Castilho EA, Andrade CLT. Mortalidade infantil no Brasil: Belíndia ou Bulgária? Cad Saúde Públ (Rio de Janeiro) 1997; 13:503-16.

3. Victora CG, Barros FC. Repensando a associação entre indicadores de saúde e de qualidade de vida. In: Costa MFFL, Sousa RP, orgs. Qualidade de vida: compromisso histórico da epidemiologia. Belo Horizonte: Coopmed - ABRASCO; 1994. p.19-23.

4. Ahmad OB, Lopez AD, Inoue M. The decline in child mortality: a reappraisal. Bull WHO 2000; 78:1175-91.

5. Claeson M, Bos ER, Mawji T, Pathmanathan I. Reducing child mortality in India in the new millennium. Bull WHO 2000; 78:1192-9.

6. Oliveira LAP, Mendes MMS. Mortalidade infantil no Brasil: uma avaliação de tendências recentes. In: Minayo MCS. Os muitos Brasis - Saúde e população na década de 80. $2^{\mathrm{a}}$ ed. São Paulo - Rio de Janeiro: Hucitec - ABRASCO; 1999. p. 291-303.

7. Puffer RR, Serrano CV. Patterns of mortality in childhood. Washington DC: PAHO; 1973 (Scientific Publication 262).

8. Victora CG, Bryce J, Fontaine O, Monasch R. Reducing deaths from diarrhoea through oral rehydration therapy. Bull WHO 2000; 78:1246-55.

9. Rutstein, SO. Factors associated with trends in infant and child mortality in developing countries during the 1990s. Bull WHO 2000; 78:1256-70.

10. Hartz ZMA, Champagne F, Leal MC, Contandriopoulos AP. Mortalidade infantil "evitável" em duas cidades do Nordeste do Brasil: indicador de qualidade do sistema de saúde local. Rev Saúde Publ 1996; 30:310-8.

11. Campos TP, Carvalho MS, Barcellos CC. Mortalidade infantil no Rio de Janeiro, Brasil: áreas de risco e trajetória dos pacientes até os serviços de saúde. Rev Panam Salud Publica 2000; 8: 164-70.

12. Rutstein DD, Berenberg W, Chalmers TC, Child CG, Fishman AP, Perrin EB. Measuring the quality of medical care: a clinical method. N Engl J Med 1976; 294:582-8.
13. Mendonça EF, Goulart EMA, Machado JAD. Confiabilidade da declaração de causa básica de mortes infantis em região metropolitana do Sudeste do Brasil. Rev Saúde Publ 1994; 28:385-91.

14. Pharoah POD, Morris JN. Postneonatal mortality. Epidemiology Review 1979; 1:170-83.

15. Saad PM. Mortalidade infantil por causas no Estado de São Paulo (Brasil) em 1983. Análise sob a perspectiva das causas múltiplas de morte. Rev Saúde Pública 1986; 20:481-8.

16. França E. Estudo de determinantes da mortalidade infantil por diarréia, pneumonia e desnutrição na Região Metropolitana de Belo Horizonte em 1991-1992 [tese]. Belo Horizonte: UFMG; 1995.

17. Niobey FML, Duchiade MP, Vasconcelos AGG, Carvalho ML, Leal MC, Valente JG. Fatores de risco para morte por pneumonia em menores de um ano em uma região metropolitana do Sudeste do Brasil. Um estudo tipo caso-controle. Rev Saúde Publ (São Paulo) 1992; 26:229-38.

18. Onis M, Frongillo EA, Blossner M. Is malnutrition declining? An analysis of changes in levels of child malnutrition since 1980. Bull WHO 2000; 78:1222-33.

19. Dean AG, Burton AH, Dicker RC. Epi Info, Version 6: a word processing, database and statistics program for epidemiology on micro-computers. Atlanta: Center for Disease Control; 1994.

20. Campos-Filho N, Franco EL. A microcomputer program for multiple logistic regression by unconditional and conditional maximum likelihood methods. Am J Epidemiol 1989; 129:439-44.

21. Viegas JMS, Dolabela RF. Estudo qualitativo das estatísticas de nascimentos e óbitos: o sub-registro em Minas Gerais e Região Metropolitana de Belo Horizonte - Resultados definitivos do Censo Demográfico de 1980. Indicadores de conjuntura 1986; 8:485-95.

22. Donabedian, A. The quality of care: how can it be assessed? JAMA $1988 ; 260: 1743-8$.

23. Claeson M, Merson MH. Global progress in control of diarrhoeal diseases. Pediatr Infect Dis J 1990; 9:345-55.

24. Black RE. Persistent diarrhea in children of developing countries. Pediatr Infect Dis J 1993; 12:751-61.

25. Schutze GE, Jacobs RF. Management of community-acquired bacterial pneumonia in hospitalized children. Pediatr Infect Dis J 1992; 11:160-4.

26. Victora CG, Barros FC, Vaughan JP. Epidemiologia da desigualdade. $1^{a}$ ed. São Paulo: Hucitec; 1988.

27. Post ALA, Victora AG, Valente JG, Leal MC, Niobey FML, Sabroza PC. Fatores prognósticos de letalidade hospitalar por diarréia ou pneumonia em menores de um ano de idade. Estudo de caso e controle. Rev Saúde Publ (São Paulo) 1992; 26:369-78.

28. Hart JT. The inverse care law. Lancet 1971; 1:405-12.

29. Puccin RF. Organização das práticas de saúde e vulnerabilidade à diarréia infantil [editorial]. J Pediatr (Rio J) 2000; 76:3-4.

30. Campos GWS. Reforma da reforma - repensando a saúde. $1^{\text {a }}$ ed. São Paulo: Hucitec; 1992.

31. Donabedian A. The seven pillars of quality. Archives of Pathology and Laboratory Medicine 1990; 114:1115-8.

32. Acurcio AF, Guimarães MDC. Acessibilidade de indivíduos infectados pelo HIV aos serviços de saúde: uma revisão de literatura. Cad Saúde Públ (Rio de Janeiro) 1996; 12:233-42.

33. Haddad, SC. Estudo dos aspectos culturais relacionados à mortalidade infantil pós-neonatal na Região Metropolitana de Belo Horizonte - 1991 a 1992 [dissertação]. Belo Horizonte: UFMG; 1999.

Endereço para correspondência:

Dr. Antônio P. Caldeira

Rua Walter Ferreira Barreto, 225 - Bairro Ibituruna

CEP 39401-347 - Montes Claros, MG

E-mail:apc@connect.com.br 